\title{
UN OBJET SCIENTIFIQUE À LA CHARNIÈRE DES SCIENCES BIOLOGIQUES ET SOCIALES: LE SYSTËME IMMUNITAIRE
}

\author{
A SCIENTIFIC OBJECT INTERLINKING \\ THE BIOLOGICAL AND SOCIAL SCIENCES: THE \\ IMMUNE SYSTEM
}

Anne Marie Moulin*

\begin{abstract}
MOULIN, A. M.: 'A scientific object interlinking the biological and social sciences: the immune system'. História, Ciências, Saúde - Manguinbos, III (2):300-318, Jul.-Oct. 1996.

Immunology gained legitimacy when it became the science of the immune system, a definition that announced the existence of a previously unknown body function. The very concept of immune system goes beyond the biological, offering opportunities for a renewed dialogue between biological science and the human sciences. This paper discusses the manifold viewpoints regarding the immune system.
\end{abstract}

KEYWORDS: anthropology, metaphor, immune system, frontier.

*Directeur de recherches CNRS

Unité INSERM 158

Hôpital Necker Paris
Un concept illustre de façon exemplaire la nouvelle donne (Kleinman, 1973) qui caractérise les relations entre sciences biologiques et médicales, d'une part, humaines et sociales, d'autre part. Ce concept est d'importance pour la nosologie, la thérapeutique comme la santé publique: il s'agit du concept d'immunité, sous la forme activée du système immunitaire. Le système immunitaire s'impose aujourd'hui comme une catégorie opératoire des sciences biomédicales contemporaines. Il n'est pas nécessaire ici de récapituler les étapes de la formation de ce concept (Moulin, 1991; Moulin et Silverstein, 1990). Je partirai de ce que je considère comme une sorte de donnée historique (Silverstein, 1989), un objet scientifique officiellement reconnu (Daëron et alii, 1996) et intégré au discours des contemporains quelle que soit leur sphère de compétence, bref sur lequel nous partageons tous un certain savoir.

\section{Le système immunitaire}

Par système immunitaire (Fridman, 1991), on entend aujourd'hui couramment un ensemble de cellules et de facteurs qui assurent une fonction dans l'organisme, appelée immunité. Immunité désignait il y a cent ans une propriété particulière liée aux 
${ }^{1}$ Voir les sens multiples du terme immunité qui apparaît une vingtaine de fois dans un texte consacré aux possibilités d'a cclimatement des Européens en Algérie (Périer, 1845). Il s'agit d'un rapport officiel évaluant les possibilités de la colonisation. phénomènes d'adaptation à des changements survenus dans le milieu, aux conditions climatiques, aux variations de la salubrité ambiante... ${ }^{1}$ Bref, une propriété accidentelle, mise en jeu dans des circonstances exceptionnelles liées au hasard d'une épidémie ou à la rencontre avec la lancette d'un vaccinateur et plus ou moins confondues avec les forces de résistance à la mort. Immunité désigne aujourd'hui une fonction permanente essentielle à la survie dans la vie quotidienne du corps. Le système immunitaire est considéré comme lié étroitement aux autres grands appareils tels que le système nerveux ou le système endocrinien.

Le système immunitaire est un fait scientifique total. Le point de vue immunologique est incontournable, quel que soit le domaine de la biologie auquel on s'adresse. L'immunologie offre désormais un axe de recherche pour tous les grands problèmes biologiques: évolution, développement, cancérogénèse, reproduction...

Le système immunitaire a attiré maintenant l'attention des sciences humaines et sociales. D'un point de vue sociologique, il a déjà rempli de véritables fonctions sociales à l'intérieur de la communauté scientifique. Il a fait converger des travaux situés dans des sphères professionnelles séparées. Il a solidarisé des recherches disparates: celles des biologistes qui travaillent en 'cis' et de ceux qui travaillent en 'trans', pour reprendre une distinction humoristique proposée par Niels Jerne en 1967 au Congrès de biologie cellulaire de Cold Spring Harbor, c'est-à-dire ceux qui travaillent sur les molécules, les produits finis de l'immunité et ceux qui explorent les mécanismes intracellulaires, autrement dit les biologistes moléculaires et les cellularistes. Plus généralement il a relié les sérologistes, les zoologistes qui travaillent à la classification des espèces, les experts de la reproduction humaine, les pathologistes, les généticiens, à qui il a fourni un dénominateur commun.

Un objet qui permet d'opérer autant de rapprochements est nécessairement un objet épistémologiquement complexe auquel va correspondre une "épistémologie fluide" (Napier, 1992; Jacquemart, 1990). Il est à la fois objet construit et objetprogramme, objet théorique et objet d'observation. Jamais la distinction entre ce qui est de l'ordre du fait, de la technique, du phénomène et de la théorie, n'est apparue aussi difficile. Peuton imaginer de faire l'économie de cette notion sans perturber gravement les possibilités de communication entre des locuteurs très divers, une de ses fonctions étant de créer des réseaux d'analogies et des possibilités d'écoute et de résonance? Notion féconde dont il devient essentiel d'explorer la richesse sémantique en restant vigilants sur les dangers introduits par la polysémie. 


\section{Sciences biologiques}

Le système immunitaire a été suggéré dans ses grandes lignes par le biologiste russe Elie Metchnikoff (mort en 1916), qui élabora sa théorie de l'immunité en s'intéressant au comportement des cellules mésodermiques dans un très grand nombre d'espèces animales. Il a interprété le rôle de ces cellules comme essentiel et bénéfique au cours des processus d'inflammation qui pour la génération de Broussais résumaient la maladie, mais au lieu de voir dans l'accumulation des cellules le stigmate et finalement la cause du processus pathologique, il l'a décrit comme un indice de la lutte de l'organisme pour se débarrasser des assaillants. Metchnikoff (1891) a nommé phagocytes ces cellules cannibales qui engouffrent les gêneurs et forment la première ébauche du système immunitaire. Les phagocytes sont de deux sortes, les cellules situées dans un organe particulier, et les cellules qui parcourent le corps entier. Metchnikoff devait subir les critiques des biologistes décelant un parfum de téléologie naturelle derrière ce langage sur les défenses de l'organisme. Sa construction théorique s'adossait de toute évidence à une philosophie prospectant les conditions d'une certaine harmonie naturelle (Tauber, 1991).

Le modèle explicatif du système immunitaire, repris et développé dans les années 60 , se proposait d'inclure toutes les vicissitudes de la vie, un système au sens profond et méme métaphysique du terme. Il était censé fonctionner comme un intégrateur du temps en ses trois dimensions, un régulateur des rapports entre les organes et l'organisme, le tout et les parties. Le modèle revêtait donc d'emblée des traits extérieurs au corpus de connaissances à l'intérieur duquel il s'était constitué et acquérait une sorte d'autonomie. Il ne renvoyait pas seulement à un objet scientifique susceptible de recevoir une traduction expérimentale au laboratoire, il pouvait être repris en compte par l'expérience subjective de la lutte contre la maladie et la mort et permettait d'interpréter la maladie comme illness autant que la maladie comme disease (Young, 1982). Le système immunitaire tendait donc à relier des réseaux de sens qui acquerraient ultérieurement de nouvelles significations.

Si les grandes lignes de la définition du système immunitaire dans les années 60 comme un ensemble de facteurs et de cellules restent aujourd'hui acceptables, de nouvelles entités ne cessent d'apparaitre dans le cadre de cette définition (Nossal, 1988), en fonction des nouvelles techniques employées. Des facteurs comme

2 Depuis l'interleukine 1 identifiée par Robert Gallo, on a atteint le numéro 15. les lymphokines sécrétées par les cellules et qui assurent des médiations à tous les niveaux ${ }^{2}$ aux cellules sélectionnées par les "cell-sorters", automates qui trient les cellules, la liste des entités 
3 Tonegawa (1987) nous donne la description de la genèse de l'étonnante diversité des anticorps, prevue aux termes de la théorie sélective de la formation des anticorps, toujours en vigueur. ne cesse de s'allonger... Les prétendus faits ne sont en définitive qu'une question de FACS, "a matter of FACS", jeu de mots intraduisible des sociologues des sciences Alberto Cambrosio et Peter Keating (1992). La métaphore de l'orchestre symphonique utilisée pour le système immunitaire dont Gershon en son temps a donné plusieurs versions successives (Moulin, op. cit.), s'accommode bien du remplacement des musiciens et même de l'éviction du chef d'orchestre ou des divisions de ses prérogatives entre plusieurs chefs de choeur. Les questions liées au système immunitaire peuvent ainsi se ramener à des problèmes d'identification fine des cellules impliquées dans les cascades de réactions immunologiques, exprimées suivant des analogies empruntées souvent à la cybernétique et aux théories de la communication: cellules et facteurs circulent et communiquent...

L'addition d'une fonction supplémentaire, celle de l'immunité, à un ensemble de fonctions déjà répertoriées, telles que nutrition, respiration, circulation... n'a été qu'un prélude à une grande première, où le système immunitaire se présentait finalement à la candidature de cause principale, à la fois formelle, matérielle et finale, pour paraphraser Aristote en matière de pathogenèse (Moulin, 1993).

Avec le système immunitaire, il ne s'agit pas de choisir entre les innombrables facteurs déjà inventoriés qui interviennent dans le développement des maladies et qui résument l'accumulation des connaissances antérieures: facteurs nutritionnels, microbiens et parasitaires, et plus près de nous, génétiques et écologiques, mais de construire un ensemble qui les contienne tous, une "trame" (Périer, op. cit.) pour reprendre le terme d'un auteur vitaliste au $19^{\mathrm{e}}$ siècle. Les facteurs parasitaires et infectieux apparaissent aisément transposables sous la forme d'anticorps ou de cellules sensibilisées, mais les troubles nutritionnels peuvent aussi être transcrits, les déficits alimentaires pouvant perturber la synthèse de certaines protéines. Les anomalies génétiques sont susceptibles de toucher la plupart des fractions du complément et des classes d'immunoglobulines. Les facteurs génétiques enfin commandent la facture même du système immunitaire dont ils règlent dans le texte la capacité combinatoire. ${ }^{3}$

Le système immunitaire est assimilable à une entité où s'éclairent les rapports de causalité qui instruisent sur la maladie, et où se projettent les temps forts d'initiation des processus pathologiques, la crise et le dénouement, une entité qui a donc une fonction étiologique, nosologique et fantasmatique, qui par conséquent sert de truchement entre les hommes de l'art de guérir et les hommes tout court. Dans les pays occidentaux, il est devenu courant d'expliquer une affection par une vicissitude du système immunitaire; il peut s'agir d'une éclipse momentanée, fatigue, 
4 Le projet Barbara Mc Clintock (1993), soutenu par l'association Act Up, a été nommé d'après la biologiste américaine Mc Clintock dont les recherches non conventionnelles sur la génétique ont été tardivement couronnées par le prix Nobel. Il vise à susciter les travaux 'alternatifs' à l'hypothèse du rétrovirus.

5 Voir, par exemple, les controverses soulevées par les critiques de Duesberg (1989, 1988) contre le rôle exclusif attribué au virus.

6

Infection et contagion n'ont pas de sens microbiologique avant la fin du $19^{*}$ siècle. grossesse, infection virale, surmenage, d'un simple incident de parcours ou d'un cataclysme comme la contamination par le virus HIV, ou même d'une malfaçon (déficit génétique). Ce qu'il y a de sûr, c'est que comme le disent les praticiens, "votre système immunitaire ne fonctionne pas bien". Il a peut-être remplacé le 'foie' des Français dans le diagnostic de la patraquerie chronique, avant d'être incriminé dans le drame de la maladie du siècle.

La puissance explicative du système immunitaire a des effets pervers. Le modèle n'est jamais à court d'hypothèses sur la pathogénie d'une affection donnée. Les hypothèses immunologiques qui permettent de reconstituer la séquence naturelle des événements restent cependant infalsifiables, et il est difficile d'apporter la preuve de leur pertinence. J'ai donné ailleurs l'exemple d'un malade atteint d'une parasitose insolite (Moulin, op. cit.). Un ver, d'ordinaire cantonné à l'intestin, se trouvait chez lui en abondance sur toute la longueur du tube digestif et avait même passé la barrière réputée infranchissable des méninges puisqu'il avait colonisé le liquide céphalo-rachidien. Les données immunologiques ne permettaient pas de décider si l'anomalie de la défense était la cause primaire de cette extension inouïe ou si la dissémination anormale du parasite était responsable d'un dysfonctionnement de l'immunité. Il était pourtant du plus haut intérêt de reconstituer l'histoire naturelle de la maladie, ne serait-ce que pour choisir la cible de la thérapeutique. Mais les différents examens ne fournissaient qu'un instantané de la situation pathologique et ne permettaient pas d'inférer l'anamnèse.

Le raisonnement physiopathologique en matière de Sida se heurte à la même difficulté. Il est difficile mais crucial de reconstituer, en amont de l'état d'immunodéficience acquise, la chaîne des événements et en particulier de déterminer si l'infection suit un cours irréversible ou si plusieurs dynamiques sont possibles. A plusieurs reprises, des tentatives de modèles alternatifs ${ }^{4}$ se sont fait jour, remettant en question la pathogénie, le rôle exclusif du virus et des réactions immunitaires en chaîne, bousculant la trame des causes et des conséquences. ${ }^{5}$ Aucun n'a réussi jusqu'à présent à supplanter le mode explicatif dominant. Une raison en est peut-étre l'inépuisabilité et l'extrême plasticité du modèle fourni par le couple du système immunitaire et de ses virus VIH. Le système immunitaire se présente comme un réseau ouvert où une forme se maintient à travers un flux d'éléments en permanente transformation et interaction.

Réseau de causes tramant logiquement de multiples facteurs, le système immunitaire permet de réinterpréter les maladies infectieuses de naguère, premier champ d'application de l'immunologie, lorsque l'infection a acquis un sens microbiologique et s'est faite spécifique. ${ }^{6}$ Il permet, aujourd'hui, à la faveur d'un 
7 Les postulats dits de Henle-Koch sont des postulats définissant à quelles conditions on peut attribuer la responsabilité d'une maladie à une bactérie. Esquissés par Henle et complétés par Koch, ils ne recouvrent pas aujourd'hui la totalité des critères exigés pour caractériser une maladie infectieuse (Carter, 1985; Huebner, 1959). Sur leur application au problème du Sida, voir Harden (winter 1989). réexamen des postulats de Henle-Koch, ${ }^{7}$ de reformuler les problématiques passées comme celles sur le rôle respectif des germes et du terrain ou celles qui se sont attachées à la notion de stress. Il est ainsi le dépositaire d'une histoire qui en module au cours du temps la signification profonde.

Prenons l'exemple du stress. On sait que cette notion, proposée par Hans Selye dans les années 30, a été forgée pour décrire les circonstances du collapsus cardio-vasculaire, le 'choc' au cours de l'expérimentation animale. Le terme de stress a désigné ensuite l'insuffisance surrénalienne aiguë succédant à la réponse physiologique à une agression, puis s'est élargi pour englober l'ensemble des réactions catastrophiques à divers traumatismes (Selye, 1980). Par un juste retour de la sémantique, stress désigne aussi aujourd'hui le degré de tension auquel est soumise une lame de métal, bref cette notion de résistance (Moulin, 1994) qu'appréhendaient confusément les vitalistes sans savoir bien la mesurer. Dans les termes mêmes de Selye (1979, 1974, 1956) qui a beaucoup contribué par ses livres pour le grand public à populariser la notion, "stress" finit par se confondre avec les accidents et les deuils, et finalement avec l'usure de la vie (Cooper, 1984), et la mort qui en indique le terme. Le stress, l'anti-résistance, s'est retrouvé dans le champ de l'immunologie et surtout de la psychoneuroimmunologie (Corson, 1988).

Autre exemple: la notion de système immunitaire n'a pas été projetée brutalement sur le devant de la scène au début de la description des premiers cas d'une maladie inconnue qui allait s'appeler le Sida. Elle se situe dans la continuité de la dynamique des idées médicales. Comme on l'a vu, le système immunitaire correspond à la maturation d'une forme parvenue à terme, caractérisée par ses possibilités d'intégration d'éléments nouveaux, causes transformables en facteurs mis en jeu par la réponse aux modifications du milieu intérieur et extérieur. Ce qui pouvait passer pour un puzzle fonctionne en fait comme une combinatoire. Cette combinatoire était disponible pour l'interprétation convergente des constats des épidémiologistes d'Atlanta et des cliniciens alertés par une pathologie inédite (Seytre, 1993). La construction scientifique du Sida aurait été différente si le système immunitaire n'avait pas fait partie de l'outillage intellectuel des années 80 .

Le meilleur exemple de la disponibilité du modèle immunologique est en effet cette période obscure où ont convergé les observations disparates d'un mal étrange, comportant en particulier des infections liées à des germes appelés "opportunistes", c'est-àdire ne déployant une pathogénicité véritable que sur terrain modifié naturellement ou artificiellement dans le cadre de la "iatrogenèse" jadis dénoncée par Ivan Illich. Le système immunitaire 
s'est trouvé disponible pour présenter les inconnues dans son algorithme. Il ne restait plus qu'à détailler les constituants du système qui sont prioritairement atteints, et qui peuvent servir d'indicateur du pronostic. On sait le rôle joué par les cellules helper T4 dans cette perspective depuis une dizaine d'années (Cambrosio et Keating, 1994).

Mais la modification du système immunitaire dans le Sida ne renvoie pas seulement aux perturbations déclenchées par les rétrovirus. Les significations du système immunitaire débordent les réactions en chaîne de classes de cellules et de molécules. La métaphore, déjà présente dans le langage médical, se déplace imperceptiblement du biologique au social, quand il devient clair que ce syndrome de déficit immunitaire atteint ceux qui sont socialement (ce qui peut se dire immunologiquement) sans défense, les fameux "groupes à risque" de nos sociétés occidentales, et finalement les continents à risque, l'Afrique et bientôt l'Asie et 1 'Amérique latine, ou l'underworld de l'Amérique du Nord.

L'immunité avait été au début du siècle conçue sous le signe de l'borror autotoxicus (Silverstein, 1986), ce qui voulait dire que l'individu ne pouvait pas s'immuniser contre ses propres constituants sous peine de mort. D’ingénieux mécanismes ont été imaginés pour faire respecter un pareil interdit par la machinerie cellulaire: la plupart sous-entendaient la neutralisation ou la destruction d'éléments aberrants apparus par suite de la capacité même de l'organisme à réagir (Ehrlich, 1901). Mais la destruction d'éléments appartenant eux-mêmes au soi faisait figure de paradoxe, portant la contradiction et par conséquent le doute dans cette superbe construction intellectuelle (Jacquemart et Coutinho, 1988). C'est en effet un paradoxe que pour respecter l'intégrité du soi, il faille aussitôt formuler l'exception en admettant l'annihilation par anticipation des éléments pouvant jouer par la suite un rôle destructeur.

L'évolution des idées s'est faite, de façon chaotique, vers l'abandon du tabou de l'autotoxicité et la reconnaissance d'une activité auto-immune. Elle a abouti dans les années 60 à la construction nosologique des "maladies auto-immunes", cadre d'abord restreint à certaines anémies inexplicables et à dessyndromes expérimentaux (encéphalites, orchites, thyroïdites) avant d'englober des affections définies dans un autre cadre nosologique (les "maladies du collagéne"), dont le lupus érythémateux est la plus représentative. La vague a atteint ensuite de nombreuses affections, parmi lesquelles le diabète et la sclérose en plaques.

Mais du même coup, l'auto-immunité pathologique apparaissait comme l'amplification regrettable d'une réaction physiologique 
8 Le modèle du soi peptidique conduit à distinguer un soi somatique et un soi immunologique (Moulin, 1990; Kourilsky et Claverie, 1988; Löwy, 1991). plutôt que comme un bouleversement du fonctionnement ordinaire de l'organisme. La théorie de l'auto-immunité généralisée (l'autoimmunité existe dans un organisme normal) a pris un sens nouveau avec les théories du réseau idiotypique (Urbain, 1977; Jerne, 1982). On s'écarte ainsi du modèle historique qui avait été celui de l'immunité anti-infectieuse où les anticorps sont avant tout une réponse antagoniste. Selon ces théories, les anticorps d'un individu, en formant un véritable réseau, interagiraient entre eux de façon permanente. Il existerait donc une auto-immunité non pas conflictuelle mais conviviale. Celle-ci pourrait jouer un rôle dans la régulation fine des réponses à l'environnement. Les réactions en chaîne du système pourraient par exemple renforcer ou abaisser le niveau d'activité des anticorps dirigés contre des molécules physiologiques appartenant à d'autres systèmes: système nerveux (c'est l'objet de la psychoneuroimmunologie [Locke et coll., 1985] qui réexamine dans cette perspective la vénérable notion de stress), système cardio-vasculaire, système endocrinien.

L'auto-immunité ne désigne donc plus un ensemble de réactions pathologiques, mais la structure même de la réactivité de l'organisme. La tentative d'énonciation d'un soi biologique, ${ }^{8}$ parce qu'elle débouche sur les apories de l'auto-immmunité, a modifié l'horizon de la recherche.

L'idée générale qui se dégage ces dernières années en immunologie est qu'il est possible de faire de la réponse immune au soi une réponse notablement différente de la réponse au nonsoi. L'immunologiste Antonio Coutinho (1989) a proposé de distinguer un système immunitaire périphérique et un système immunitaire central caractérisé par une connectivité élevée et une multiréactivité. Le fonctionnement du premier est le fonctionnement immunitaire classique, élaboration d'une réponse clonale et spécifique aux agressions de l'environnement. Le fonctionnement du second est systémique et présente un régime oscillant, voire parfois chaotique. La reconnaissance du soi n'est pas seulement un phénomène négatif qui s'exprime par des activités de suppression, mais par une activité positive. En cas de maladie, la frontière entre ces deux systèmes immunitaire, normal et périphérique, perdrait de sa distinction. Les deux régimes s'homogénéisent, et le système immunitaire central tend alors à fonctionner selon un mode clonal qui ne lui est pas habituel, entraînant des dégâts sur les cibles des anticorps en expansion. La thérapeutique qui en découle logiquement est l'introduction d'autoanticorps naturels multiréactifs susceptibles de rétablir le régime normal et la concertation harmonieuse du systéme.

De telles spéculations sont une source potentielle d'innovations thérapeutiques. De façon très générale, il devient théoriquement 
9 Dans les années 70 , l'hématologue Maxime Seligmann avait proposé en France (sans succès) un laboratoire d'immunologie comme trone de réflexion commun (et non plus comme lieu de production d'examens "complémentaires" spécialisés) à tous les départements de médecine. possible de manipuler le système immunitaire en introduisant dans l'organisme des anticorps dirigés contre certains motifs moléculaires du soi, dits encore motifs idiotypiques, et de déclencher des perturbations aux fins de rétablir un régime normal. Toute maladie n'est pas nécessairement exprimable en termes d'immunité, mais pourrait étre manipulable immunologiquement. Devient ainsi concevable par exemple une thérapeutique immunologique de l'hypertension artérielle qui agisse sur les anticorps antirénine, la rénine étant un enzyme impliqué dans la libération de peptides doués d'une puissante action vasopressive, qui a été incriminé dans le déclenchement des hypertensions permanentes. On a pu proposer un anticorps monoclonal pour prévenir le choc septique, le cauchemar des réanimateurs. Au terme général d'immunomodulation qui tend à se substituer au couple immunosuppression/immunostimulation, répond le terme immunosomatique qui coiffe toutes les spécialités médicales.?

\section{Sciences humaines}

Le système immunitaire ne fonctionne donc pas seulement comme une catégorie physiologique descriptive, mais comme une catégorie herméneutique d'une portée plus générale. Il s'agit bien, à l'occasion d'un développement apparemment interne aux sciences biologiques, de la constitution d'un nouveau champ de signes (Augé et Herzlich, 1980). L'individu et son environnement, l'individu et son histoire, le rapport entre santé personnelle et santé publique y sont déchiffrables (Nichter, 1992).

Un vidéo-clip utilisé pour présenter les objets de la recherche scientifique et intitulé Le système immunitaire illustre parfaitement la perméabilité des frontières de sens entre sciences biologiques et sciences humaines ou même entre science et littérature. On y suit avec émotion la transcription du système immunitaire opérée par une mère attentionnée déchiffrant avec ses mots à elle l'objet des savantes recherches de son fils:

"Je ne te dirai pas le bonheur que tu me donnes, tu m'entrouvres ton jardin." Jardin est la métaphore choisie pour désigner le monde secret de son fils. Mais c'est encore la métaphore qui lui vient pour parler de l'objet même de ses recherches. A propos du système immunitaire: "Je ne peux m'empêcher de penser à un jardin, avec ses massifs, allées et bordures." Ou encore, plus loin: "Je le vois (toujours le système) comme un gros livre où le grand-père consignait les événements: il est difficile de repérer des changements si nous ne sommes faits que de cela."

La découverte de la réversibilité du monde extérieur et de l'intériorité de l'organisme (Daëron, 1988) se fait tout naturellement: "Nous sommes grand ouverts au monde extérieur: 
Scénario dû à Marc Daeron, Laboratoire d'mmunologie cellulaire et clinique, INSERM U 255, Institut Curie, Paris.

11 Voir, par exemple, l'analyse du stress (Young, 1980).

12 Il faut rappeler ici le rôle pionnier de Frantz Fanon, interprétant la perception de la médecine coloniale pendant la guerre d'Algérie en termes d'agression psychologique nous sommes l'extérieur pour une foule de microbes: serait-ce que le milieu extérieur ne nous est pas totalement étranger?"

L'intériorisation de l'auto-immunité est aussi tranquillement perçue dans une intuition familière. "Le système veille à nous garder nous-mêmes, je le vois." Il n'a d'autre choix que de "comparer ce qui est avec ce qui était".

Ce clip ${ }^{10}$ simple et émouvant, poursuivant un but de vulgarisation par les voies inédites de la méditation poétique, sert à merveille mon propos: le système immunitaire, création officielle de l'immunologie contemporaine, illustre les parentés souterraines des sciences biologiques et humaines. Il puise à cet inconscient dont Bachelard souhaitait que nous explorions les sources et le potentiel créateur, au nom même de la rationalité.

Les anthropologues ont été les premiers à analyser la concordance entre ce système de signes et les autres objets théoriques produits par notre culture. ${ }^{11}$ Donna Haraway (1989) voit dans le système immunitaire une "icône post-moderne" caractéristique de la transformation du corps, sain ou malade, en un robot évaluable et manipulable par le pouvoir médical ou ses succédanés. Les campagnes d'inoculation, dont on connait le rôle paradigmatique dans l'histoire de la médicalisation, n'ont été que le prélude d'une mise au point des immunisations de masse. Le système lui-même est perçu comme une machinerie installée à l'intérieur de la personne et dont celle-ci est en train de perdre le contrôle au profit d'instances extérieures. L'écriture agressive de ces manipulations, de cette mise au pas du corps individuel et collectif, est perçue par Donna Haraway comme un stigmate caractéristique de la science moderne, science machiste, incapable de penser autrement qu'en métaphores dualistes, mécanistes et guerrières que dénonce en d'autres domaines Evelyn Fox-Keller (1995; Napier, 1996).

Les historiens eux-mêmes ${ }^{12}$ qui ont subi l'influence de Foucault, Surveiller et punir, tout en respectant le bien-fondé et les bienfaits démographiques résultant des stratégies de santé publique, sont sensibles aux connotations punitives et théologiques des campagnes d'immunisation, au bouleversement des solidarités traditionnelles et des genres de vie et aux inégalités subrepticement renforcées (Rivet, 1995; Peter, 1989; Weindling, 1993).

Analysant les réactions de la population américaine aux informations sur le Sida, une autre anthropologue, Emily Martin (1994, 1989), voit aussi pour d'autres raisons dans le système immunitaire un produit typique de l'idéologie de notre temps. Le système immunitaire de première génération, celui des années 60 , était conçu comme un organe central (pourquoi pas le thymus?), avec des relais en périphérie et des voies centrifuges et centripètes. Il en va différemment de la dernière version du 
13 Fainzang (1992). Pour les problèmes textuels liés aux questions de commensurabilité, voir Zimmermann (1989). système: elle correspond aux nouvelles exigences de notre société: mobilité, disponibilité, polyvalence (idem, 1990). La transcription de ces changements d'orientation est sensible dans les dernières représentations du système immunitaire, marquées par la décentralisation et l'abandon de la hiérarchisation des organes, le refus du point de vue clonal, la réélaboration de la notion de spécificité. Bref, le système lu au revers permettrait de déchiffrer les nouveaux mots de passe: plasticité, disponibilité, polyvalence, décentralisation. Est-il besoin d'insister sur le fait qu'il s'agit des impératifs auxquels est soumis le citoyen américain sur le marché de l'emploi, et qu'Emily Martin redécouvre une Weltanschauung qui gouverne également les rêves des puissants et des officiels et leurs nouveaux lettrés? Le système immunitaire est adéquatement décrit comme un "cerveau mobile", selon l'expression choisie par Hervé Fridman (op. cit.) dans sa présentation au grand public de l'immunologie contemporaine.

\section{Anthropologie médicale}

Ce dernier exemple montre bien que le concept de système immunitaire fonctionne métaphoriquement et ouvre des voies de communication inédites. Les métaphores guerrières et anthropomorphiques ne sont pas à pourchasser systématiquement, mais à observer attentivement, puisque la métaphoricité de la science n'est pas réductible comme une interprétation chagrine de la science pourrait le faire croire. Cette métaphoricité est la clé de la signifiance anthropologique des concepts scientifiques. Bref, le système immunitaire joue le rôle de référent anthropologique au sens large.

Du coup, il apparaît utilisable comme une 'sonde' ou un 'marqueur' pour explorer les différentes interprétations culturelles de la maladie, qu'il s'agisse de suivre la réception des idées occidentales concernant la pathogénése du Sida, ou de tenter une analyse comparée (Kleinman, 1987) entre des visions devenues commensurables. ${ }^{13}$ Le système immunitaire correspond à un codage particulier de notre corps, enraciné dans le savoir scientifique et la culture d'une époque. Mais le champ de signes qu'il a ouvert est transposable à d'autres périodes du passé comme à des cultures différentes, introduisant une parenté entre des formes de langage de la maladie apparemment éloignées (Zimmermann, 1995).

Dans cet ordre d'idées, l'anthropologue américain David Napier a lancé un programme d'étude des réactions au savoir occidental du Sida qui diffuse dans différentes cultures: comment la maladie et son substrat, le système immunitaire, sont-ils compris et réinterprétés? Comment les Indonésiens de Bali, par exemple, 
peuvent-ils l'intégrer à leur démonologie quotidienne? Ailleurs, on sait coment les Japonais ont non seulement intégré la vaccination antivariolique à leurs anciens rituels contre la variole, mais représentent encore leurs kami, divinités protectrices contre des maux spécifiques (imparfaitement traduits comme les anges gardiens), comme jouant un rôle dans la défense contre le virus modernisé de la variole (Rotermund, 1991).

Le système immunitaire joue le rôle de barrière et de limite (Moulin, à paraître) vis-à-vis du monde extérieur et comme tel se prête à toutes sortes d'interprétations qui rappellent la place énigmatique du corps dans la nature, source de puissantes métaphores. Il attire en même temps l'attention sur le caractère relatif de ces notions de barrière et de limite, éminemment mobilisables (Zinkernagel, 1996). On a vu comment la problématique de l'auto-immunité ouvrait une source de questionnement sur les limites du soi et l'impossibilité d'en donner une définition autre que fonctionnelle. De même les opérations de transfusion et de transplantation ont abouti à réouvrir la question de l'altérité. Le travail d'identification biologique sur les rapports de l'homme et de ses congénères a croisé une interrogation douloureuse sur la solidarité de la société et même sur l'existence problématique d'une communauté entre les vivants et les morts (Moulin, 1995, 1993).

Si le système immunitaire revêt la fonction épistémologique de marquer les limites entre nature et culture, entre soi et autrui, entre le vivant et le mort, il n'est plus seulement pertinent d'observer la réception du "système immunitaire" dans d'autres cultures, il est loisible d'en déchiffrer immédiatement les analogues dans ces cultures.

Chez les populations Ngbaka de Centrafrique, la maladie est rapportée aussi bien à l'agression de l'extérieur: mouches, moustiques, mélipones minuscules qui envahissent les yeux, le nez, les oreilles, jusqu'au coucher du soleil quand on se déplace en forêt, qu'à la présence d'un animal dévoreur présent dans le corps qu'il anime. Les agissements de cette bête qui se nourrit et

14 C'est ainsi que l'aménorrhée comme les métrorragies trouvent un mode facile d'explication. La normalité, les menstrues régulières, nécessaires à la fécondité, correspondant à une courte période où l'animal se nourrit, illustrent bien l'ambivalence de ce parasite indispensable à la vie (Moulin, 1983; Pagézy, 1984). nourrit son hôte, le corps, sont à la fois essentiels à la vie qu'ils manifestent et responsables de toute la pathologie présentée selon des figures d'oppositions comme grossir/dépérir; saigner/ ne pas saigner, ${ }^{14}$ écoulement/rétention des différents liquides organiques... etc. Cet animal dévoreur, fonctionnant sur le mode convivial ou destructeur, n'est-ce pas une figure du soi à soi, et du moi à l'autre, où dissociation et fusion alternent naturellement?

Chez les Avikam de Côte d'Ivoire (Gely, 1991), la survenue d'une plaie cutanée minime est vécue dramatiquement comme une ouverture supplémentaire qui perturbe gravement les flux normaux entre le milieu et l'individu. Une atteinte insignifiante, 
15 Champignons comme Candida, Aspergillus, Cryptocoques, Algues... en bouleversant un équilibre, est la porte ouverte à l'inversion des flux, l'homogénéisation du milieu, la mort... Mais l'immunologiste anglais Gell (1980) ne s'exprimait pas autrement lorsqu'il parlait du système immunitaire qui, lorsqu'il se voit élaguer ou retrancher une branche, risque de "saigner à mort". Ou que dire des incertitudes de la vaccinologie lorsqu'elle s'interroge sur les effets de vaccinations précoces comme le vaccin antirougeoleux sur la mortalité infantile générale en milieu tropical ou le vaccin antipaludéen le jour où il sera disponible sur une large échelle? (Moulin, 1995).

Pour revenir en Europe, l'intervention chirurgicale était jadis magnifiée comme un des points d'orgue de la médecine moderne, l'aboutissement de l'entreprise cartésienne de réparation des organes. Cette vision optimiste subit aujourd'hui des retouches. L'habileté du chirurgien démiurge et la haute technicité de ses gestes sont encore célébrées, d'autant que la spécialisation croissante affine les interventions dans de nombreux domaines: à preuve les progrès de l'orthopédie reconstructive ou de la neurochirurgie d'exérèse. Mais parallèlement se fait plus aiguë et parmi les chirurgiens eux-mêmes, la conscience que la chirurgie est un traumatisme. La réanimation qu'on définissait comme surveillance et rétablissement des fonctions physiologiques est un ensemble de techniques non naturelles ou même contre-nature, dont la prolongation est à la fois cause et conséquence de désordres organiques, notamment immunologiques. Les infections à germes multirésistants dits hospitaliers ont été le premier signal d'alarme, l'attention se porte maintenant vers la multiplication des infections qui ont pour point de départ des germes ou parasites ${ }^{15}$ hébergés ordinairement par notre corps, dont la vie multiple explose.

Même évolution pour la dialyse rénale, alternative toujours possible à la transplantation d'organes. Elle n'apparaît plus comme la suppléance quasi illimitée dans le temps d'une fonction défaillante mais comme une relance de la machine au prix d'une altération croissante de ses rouages. Le dialysé est un homme recomposé et peut-être dénaturé.

Le système immunitaire apparait donc ainsi comme la façon dont les médecins expriment le rapport entre le rétablissement naturel de la santé, la dynamique spontanée du vivant et l'intervention thérapeutique. L'occasion idéale pour une réflexion lucide sur le prix à payer pour les interventions humaines comprises comme déplacement d'un équilibre. Si on oppose habituellement les systèmes de représentation de la maladie dans les cultures afro-asiatiques comme liés à la recherche des causes surnaturelles, ou à un désordre social, à la malédiction ou la mauvaise volonté d'un des membres de la société, on voit qu'un rapprochement s'esquisse avec la mise en cause dans nos cultures 
${ }^{16}$ Ce qui ne revient pas à établır une continuité entre les différentes traditıons et systèmes méchcaux (Zimmermann, 1978)

17 Voir la présentation de loeuvre immunologique d'Antonio Coutinho par le joumal Libération sous le tute 'Au sein de la biologie, l'immunologie est une véritable école de tolérance', illustrée par le portrait cle Coutinho tenant clans la même main une souris blanche et une souris noire (Libéralion, 22 Mai 1991, p. 20). occidentales de la responsabilité humaine dans la pathologie, par exemple à propos de la diffusion de l'épidémie de Sida. La présentation de ce rôle a souvent été caricaturale, à propos par exemple du changement des moeurs sexuelles ou de la licence attribuée à certaines tribus africaines. Mais on peut également percevoir un écho accusateur dans certains commentaires sur les contaminations par transfusion. L'extension des transfusions de confort en chirurgie, la banalisation de l'usage des concentrés antihémophiliques ont joué un tôle dans l'inflation de l'épidémie, où la responsabilité humaine est prise à partie.

Le système immunitaire soulève donc des questions proprement anthropologiques. ${ }^{16} \mathrm{C}^{\prime} e s t$ une catégorie de la médecine occidentale, mais qui peut se déplacer, se transférer, se modifier, bref à laquelle on peut appliquer les tropes disponibles dans nos langues (Brandt, 1988); c'est aussi une catégorie qui s'impose à la fois aux malades et aux médecins (Del Vecchio-Good et alii, 1988) et rétablit une communication distendue par l'évolution des techniques et des savoirs. ${ }^{17}$

\section{Conclusion: une phénoménologie de notre temps}

A travers ces dépaysements qui fournissent une phénoménologie insolite, on peut revenir désormais sur le système immunitaire, tel qu'il a été proposé par les biologistes et le percevoir comme un rapport original de l'être au monde. Il est loisible de reconstruire l'essence de cette visée originale, qui associe une visée cognitive (déchiffrement, reconnaissance, identification...) à des opérations destructives. Si maintenant par une "réduction" (epochê) familière à la phénoménologie husserlienne, on suspend son jugement sur l'existence et la réalité du monde extérieur, on peut encore ressaisir dans le système immunitaire, un équivalent ou une "image" du monde, terme qui a été employé avec succès par Niels Jerne (1974) dans un article célèbre.

Plus fondamentalement, le système immunitaire ne peut-il pas lui-même être analysé comme l'archétype de la question, centrale aujourd'hui dans l'anthropologie occidentale, de la place mutuelle du soi et de l'autre (Fabian, 1990, 1983), place variable par ailleurs en fonction des cultures (Pouillon, 1989, 1970).

$\mathrm{Si}$, dans les premières versions du système immunitaire, l'Autre était envisagé comme l'ennemi, dans les versions plus récentes come celle du système idiotypique, l'Autre peut être perçu à travers la médiation de l'univers constitué par les antigènes du soi, sans contact direct avec l'extérieur.

La dernière version du système immunitaire est une version solipsiste où l'organisme peut explorer la totalité de l'univers sans sortir de soi. Le système immunitaire peut donc servir d'illustration 
à la difficulté majeure du savoir occidental à faire face à l'Autre, à le reconnaître et à se situer par rapport à lui. La crise se marque par le refus actuel des catégories de tropical, d'exotique, d'orientalisme. Le système immunitaire figure ainsi la difficulté majeure de l'anthropologie occidentale, la rencontre de l'autre.

Le système immunitaire est donc un concept scientifique indissolublement témoin de la métaphoricité de la science. Que les modèles mathématiques se succèdent pour améliorer son graphisme ne saurait dissimuler son fondement anthropologique, qui le rend du même coup utilisable comme un instrument d'analyse par les sciences humaines.

MOULIN, A. M.: 'Um objeto científico; a articulação das ciências biológicas e sociais: o sistema imunológico'. História, Ciências, Saĩde - Manguinhos, III (2):300-318, jul.-oct. 1996.

A imunologia legitimou-se ao tornar-se a ciência do sistema imunológico - esta definição marcou a existência de uma nova função até então desconhecida entre as funções corporais - o próprio conceito de sistema imunológico ultrapassa o biológico e possibilita o estabelecimento de um diálogo específico entre a ciência biológica e as ciências humanas. Este artigo trata das inúmeras versões existentes sobre o sistema imunológico.

PALAVRAS-CHAVE: antropologia, metáfora, sistema imunológico, fronteira.

\section{RÉFÉRENCES BIBLIOGRAPHIQUES}

Augé, M. et Herzlich, Le sens du mal, Paris.

C. (éds.)

1980

Brandt, A. 1988

Cambrosio, A. et

Keating, P. 1994

Cambrosio, A. et Keating, P. 1992

Carter, K. C. 1985

Cooper, E. L. (éd.) 1984

Corson, S. A. 1988

Coutinho, A. 1989

Daëron, M. et alii. 1996

'Aids and metaphor; toward the social meaning of social diseases'. Social Research, 55: 413-32.

'Ours is an engineering approach: flow cytometry and the constitution of human T-cell subsets'. Joumal of the History of Biology, 27: 449-79.

'A matter of FACS: constituting novel entities in immunology'. Medical Anthropology Quarterly, 6: 362-84.

'Koch's postulates in relation to the work of Jacob Henle and Edwin Klebs'. Medical History, 29: 353-74.

Stress, immunity and aging. New York, Marcel Dekker.

'Historical and philosophical background of immunoneuromodulation'. International Joumal of Neurosciences, 39: 283-7.

'Beyond clonal selection and network'. Immunological Reviews, 110: 66-87.

Le système immunitaire ou l'immunité cent ans après Pasteur. Paris, Nathan. 
Daëron, $M$. 1988

Del Vecchio-Good, M. J. et alii. 1988

Duesberg, P. 1989

Duesberg, $\mathrm{P}$. 1988

Ehrlich, P. 1901

Fabian, J. 1990

Fabian, J. 1983

Fainzang, S. 1992

Fox-Keller, E. 1995

Fridman, W. H. 1991

Gell, P. G. 1980

Gely, M. 1991

Haraway, D. 1989

Harden, V. winter 1989

Huebner, R. J. 1959

Jacquemart, F. 1990

Jacquemart, F. et Coutinho, A. 1988

Jerne, N. K. et alii. 1982

Jerne, N. K. 1974

Jerne, N. K. 1967

Kleinman, A. 1978

Kleinman, A. M. 1973
'Connais-toi toi même'. Colloque de Cérizy Praxis et Cognition.

'Emotion, illness and healing in Middle Eastern'.

Culture, Medicine and Psychiatry, 12, 1: 1-136.

'Human immunodeficiency virus and acquired immunodeficiency syndrom. Correlation but not causation'. Proceedings of the National Academy of the USA, 86: 755-64.

'HIV is not the cause of Aids'. Science, 241: 514-6.

Berliner Klinische Wochenschrift, 28: 251.

'Presence and representing the other and anthropological inquiry'. Critical Inquiry, 16: 753-72.

Time and the other. How anthropology makes its object.

New York, Columbia University Press.

'Réflexions anthropologiques sur la notion de prévention'. In P. Aïach, N. Bon et P. Deschamps, Comportments et santé. Questions pour la prévention. Nancy, Presses Universitaires de Nancy, pp. 18-27.

Refiguring life: metaphors of twentieth century biology.

New York, Columbia University Press.

Le cerveau mobile.

Paris, Hermann, p. 15.

'Network concepts in science and the arts'. The immune system, a festschrift in bonor of Niels KajJerne. Basel, S. Karger, p. 58.

'Le corps fragile: écologie du corps et syncrétisme médical chez les Avikam lagunaires de Côte d'Ivoire'. Sciences Sociales et Santé, 9: 5.

'The biopolitics of post-modern bodies: determination of self in the immune system discourse'. Joumal of Feminist Cultural Studies, 11: 3-43.

'Koch's postulates and the etiology of Aids.

An historical perspective'. Daedalus.

'The virologist's dilemma'. Annals of the New York Academy of Sciencs, 67: 430-8.

Préliminaires à une théorie gênérale anthropocentriste des objets mous. Thèse de doctorat en immunologie. Université de Paris 6.

'Observer, immune system and their respective objects'. In E. E. Sercarz and coll., The semiotics of cellular communication in the immune system. Nato Asi Series H, Springer Verlag, 23, p. 173.

'Recurrent idiotypes and internal images'.

EMBO Joumal, 1: 243.

'Towards a network theory of the immune system'. Annales d'Immunologic (Institut Pasteur), 125C: 373.

'Waiting for the end'. Cold Spring Harbor Symposia for Quantitative Biology, 32: 569-75.

'Concepts as a model for the comparison of medical systems and cultural systems'. Social Sciences and Medicine, 12: 85-93.

'Medicine's symbolic reality. On a central problem in the philosophy of medicine'. Inquiry, 16: 206-13 
Kourilsky, P. et

Claverie, J. M. 1988

Locke, A.; Ader,

R. and coll. 1985

Löwy, I. 1991

Martin, E. 1994

Martin, E. 1990

Martin, E. 1989

Metchnikoff, E. 1891

Moulin, A. M. 1997

Moulin, A. M. 1995

Moulin, A. M. 1995

Moulin, A. M. mars. 1994

Moulin, A. M. 1993

Moulin, A. M. 1993

Moulin, A. M. 1991

Moulin, A. M. 1990

Moulin, A. M 1990

Moulin, A. M. 1983

Napier, A. D.

Napier, A. D. 1996

Napier, A. D. 1992

Nichter, $M$. 1992
'Le modèle du soi peptidique'.

Médecine/Sciences, 4: 177-83.

Foundations of Psycboneuroimmunology.

New York, Aldine.

'The immunological construction of the self'. In A. I. Tauber, Organism and the origins of self. Boston, Kluwer, pp. 43-75.

Flexible bodies: tracking immunity in American culture from the days of polio to the age of Aids. Boston, Beacon.

'Toward an anthropology of immunology: the body as nation state'. Medical Antbropology Quarterly, 4: 410-26.

'The cultural construction of gendered bodies: biology and metaphors of production and destruction'. Ethos, 54: 143-60.

Leçons sur la pathologie comparée de l'inflammation.

Paris, Masson.

'Une science dans le siècle. immunology or the science of Boundaries'. In John Krige et D. Pestre (éds.), Science in the twentieth century. Amsterdam, Harwood Academic Publishers.

'Hasard et rationalité dans l'approche vaccinale'. History and Pbilosopby of Life Sciences, 17: 5-30.

'La crise éthique de la transplantation d'organes.

À la recherche de la compatibilité culturelle'. Diogène, 172: 76-96.

'Un devise pour l'organisme'. Autrement, numéro spécial Résister, pp. 22-36.

'Body parts: the modern dilemma'. Transplantation Reviews, 95: 33-55.

'The dilemma of medical causality and the issue of biological individuality'. In C. Deleskamp-Hayes et M. A. Gardell Cutter, Science, technology and the art of medicine: European-American dialogues. Dordrecht, Reidel, pp. 153-64

Le dernier langage de la médecine. Histoire de l'immunologie de Pasteur au Sida. Paris, Presses Universitaires de France.

'La métaphore du soi et le tabou de l'auto-immunité'. In M. Bessis, J. Bernard et C. Debru (éds.), Le soi et le non-soi. Paris, Seuil, pp. 55-68.

(avec A. M. Silverstein). In J. J. Oppenheim et E. Schevach (éds.),

Immunophysiology. The role of cells and cytokins in immunity and inflammation. Oxfor, Oxford University Press, pp. 3-13.

'Lévi-Straus à Kaka (République centrafricaine)'.

Bangui, rapport CNRS.

The age of immunology. (forthcoming)

'Penser vaccinologiquement: une sélection qui n'est pas vraiment naturelle, ou les modèles sociaux du monde microbien'. In A. M. Moulin (éd.), L'aventure de la vaccination. Fayard, Paris, pp. 409-22.

Foreign bodies: performance, art and symbolic anthropology.

Berkeley, California University Press.

'Of ticks, kings and the promise of vaccines'. In C. Leslie et A. Young (éds.), Patbs to Asian, medical knowledge. Berkeley, University of California Press, pp. 224-56. 
Nossal, G. B. 1988

Pagézy, H.; Couillot, M. F. et Moulin, A. M. 1984

Peter, J.-P. 1989

Périer, J. N. 1845

Pouillon, J. 1989

Pouillon, J. 1970

Rivet, $\mathrm{D}$. 1995

Rotermund, H. O. 1991

Selye, H.

Selye, $\mathrm{H}$. 1979

Selye, $\mathrm{H}$. 1974

Selye, $\mathrm{H}$. 1956

Seytre, B. 1993

Silverstein, A. M. 1989

Silverstein, A. M. 1986

Tauber, A. I. 1991

Tonegawa, S. 1983

Urbain, J. and coll. 1977

Weindling, P. 1993

Young, A. 1982
'Trials and triumphs of immunology in the 1980s'. Immunology Today, 9: 286-91.

Enquête ethnographique sur la grossesse et l'allaitement, examens médicaux et considérations ethnomédicales. République Centrafricaine, Paris, rapport CNRS.

'Dimensions mysthiques des épidémies et Sida'.

Action et recherches sociales, 3: 15-29.

'De l'acclimatement en Algérie'. Annales d'hygiène publique et de médecine légale, 33: 301-38.

In M. Segalen (éd.), L'autre et le semblable. Regards sur l'ethnologie des sociétés contemporaines. Paris, Presses du CNRS.

'Malade et médecin: le même et/ou l'autre? (Remarques ethnologiques)'. Nownelle Revuc de Psychanalyse, 1: 78-98.

'Hygiénisme colonial et médicalisation de la société marocaine au temps du protectorat français'. In E. Longuenesse (éd.), Santé, médecine et sociêté dans le monde arabe. Paris, L'Harmattan, pp. 105-28.

Hôsôgami ou la petite vérole aisément.

Paris, Maisonneuve et Larose.

'The development of the stress concept'. In H. Selye et S. Parvez (éds.), Advances in experimental medicine A centenary tribute to $C$. Bernard. Elsevier, North Holland Biomedical Press, pp. 43-69.

The stress of my life. New York, Van Nostrand Reinhold.

Stress without distress. New York, Lippincott.

The stress of life. New York, McGraw Hill.

Sida, les secrets d'une polémique. Paris, Presses Universitaires de France.

A bistory of immunology. New York, Academic Press.

Cellular immunology, 97: 173-88.

Metchnikoff and the origins of immunology. From metaphor to theory. New York, Oxford University Press.

'Somatic generation of antibody diversity'. Nature, 302: 5, pp. 9-15.

'Idiotypic regulation of the immune system by the induction of antibodies against anti-idiotypic antibodies'. Proceedings of the National Academy of Sciences of the USA, 74: 5,126

'Medicine and holocaust. The case of typhus'. In I. Löwy (éd.), Medicine and change: historical and sociological studies of medical innovation. Montrouge, INSERM/John Libbey, pp. 447-64.

'The anthropology of illness and disease'.

Annual Review of Anthropology, 11: 257-85. 
Young, A.

1980

Zimmermann, $\mathrm{F}$. 1995

Zimmermann, F. 1989

Zimmermann, F. 1978

Zinkernagel, R. M. 1996
'The discourse on stress and the reproduction of conventional knowledge'. Social Sciences and Medicine, 14B: 133-46.

Généalogie des médecines douces. Paris, PUF.

'Terminological problems in the process of editing and translating in sanskrit medical texts'. Approaches to traditional Chinese medical literature. Kluwer, P. O. Unschuld éd., pp. 141-50.

'From classic texts to learned practice: methodological remarks on the study of Indian medicine'. Social Sciences and Medicine, 12: 97-103.

'Immunology taught by viruses'.

Science, 271: 173-8.

Recebido para publicação em fevereiro de 1996. 